\section{Consideraciones en el tratamiento ortodóncico de pacientes con necesidades especiales. Revisión de la literatura}

\section{Considerations in the orthodontic treatment of patients with special needs. Review of the literature}

\begin{abstract}
Resumen
Las necesidades especiales en salud han sido definidas por la Asociación Americana de Odontopediatría como "toda condición o limitación física, del desarrollo, mental, sensorial, conductual, cognitiva o deterioro emocional que requiere tratamiento médico, intervención de atención de la salud, y/o el uso de servicios o programas especializados". Actualmente, los pacientes con necesidades especiales en salud presentan una mayor tasa de supervivencia y expectativas de vida mayores. Además, presentan una mayor prevalencia y severidad de anomalías dento-maxilares que impactan negativamente su salud general y calidad de vida. La creciente preocupación de los padres y profesionales por el aspecto estético y funcional, ha llevado a un aumento en la demanda por tratamiento de ortodoncia, sin embargo, el acceso a él sigue siendo limitado. El objetivo de la presente revisión es describir según la literatura disponible, las consideraciones para el tratamiento ortodóncico en pacientes con necesidades especiales en salud. Se concluye que el tratamiento no debe ser denegado solo por presentar una discapacidad; no obstante, el compromiso de los padres y/o cuidadores con el tratamiento es crucial para el éxito. El tratamiento debe ser planificado en etapas, siendo la fase de adaptación a la atención de gran importancia. La longitud de tratamiento en estos pacientes, es similiar a la de individuos sanos, pero se requiere un mayor tiempo-sillón y los resultados obtenidos suelen ser inferiores. El tratamiento de ortodoncia puede mejorar la estética y función en pacientes con situación de discapacidad, facilitando su integración social e impactando positivamente en su calidad de vida.
\end{abstract}

Palabras clave: Atención dental para personas con discapacidades; Maloclusión; Ortodoncia (fuente: DeCS BIREME).

\begin{abstract}
The American Academy of Pediatric Dentistry defines Special Health Care Needs as "any physical, developmental, mental, sensory, behavioral, cognitive, or emotional impairment condition or limitation that requires medical management, health care intervention, and/or the use of specialized services or programs". Currently, patients with special
\end{abstract}

\section{Artículo de Revisión}

Noemí Leiva-Villagra 1,a,b Francisca Ayala-Jiménez ${ }^{1, a}$, Carolina Stange-Dempster ${ }^{1, a}$, Vanessa Fuentes-Flores ${ }^{1, a}$, Sebastián Véliz-Méndez ${ }^{1, a, b}$

${ }^{1}$ Universidad de Chile, Facultad de Odontología, Unidad de Malformación Cráneofacial. Santiago, Chile.

a Cirujano Dentista.

${ }^{\mathrm{b}}$ Especialista en Ortodoncia y Ortopedia Maxilofacial.

\section{Correspondencia:}

Noemí Leiva

Correo electrónico: leivanoemi@yahoo.com

General Holley 2381, Providencia. Santiago, Chile.

\section{Coautores: \\ Francisca Ayala \\ francisca@ayalajimenez.com \\ Carolina Stange \\ carostange@hotmail.com \\ Vanessa Fuentes \\ vane.fuentes.f@gmail.com \\ Sebastián Véliz \\ sebastianvelizm@gmail.com}

\section{Editor invitado:}

Juan Carlos Cuevas-González

Universidad Autónoma de Ciudad Juárez, México.

Conflicto de intereses: Los autores declaran no tener conflictos de interés.

Fuente de financiamiento: Autofinanciado.

Recibido: 27/05/19

Aceptado: $10 / 07 / 19$

Publicado: $27 / 11 / 19$ 
health needs present a higher survival rate and higher life expectancies. In addition, they present a higher prevalence and severity of malocclusion that negatively impact their general health and quality of life. The growing concern of parents and professionals for the aesthetic and functional aspect, has led to an increase in the demand for orthodontic treatment, however, the health care access still remains limited. A review of the available literature was performed aiming at describing the considerations in the orthodontic treatment of special health care needs patients. It is concluded that the treatment should not be denied just for presenting a disability. Notwithstanding, it is crucial for the treatment success parents and/or caregivers commitment. The treatment should be planned in stages, being of great importance the care adaptation phase. The overall treatment time is similar to those patients without special needs, but still requires longer armchair time and the results obtained are usually lower. Orthodontic treatment can improve aesthetics and function in patients with disabilities, facilitating their social integration and positively impact in their quality of life.

Keywords: Dental care for disabled; Malocclusion; Orthodontics (source: MeSH NLM).

\section{Introducción}

Las Necesidades Especiales en Salud (NES) han sido definidas por la Asociación Americana de Odontopediatría (AAPD por sus siglas en inglés) como "toda condición o limitación física, del desarrollo, mental, sensorial, conductual, cognitiva o deterioro emocional que requiere tratamiento médico, intervención de atención de la salud, y/o el uso de servicios o programas especializados. La condición puede ser congénita, del desarrollo o adquirida a través de enfermedad, traumatismo o causa medio-ambiental y puede imponer limitaciones en la realización de actividades de auto-mantenimiento diario o limitaciones sustanciales en una actividad importante de la vida. La atención de salud para las personas con necesidades especiales requiere de conocimiento especializado adquirido por entrenamiento adicional, así como de preocupación, dedicación, manejo y adaptación de técnicas más allá de lo que se considera de rutina" ${ }^{1}$.

Chile ha experimentado cambios demográficos importantes durante las últimas décadas, presentando una disminución en la mortalidad infantil comparable con países desarrollados $(7,4 / 1000 \text { nacidos vivos })^{2}$. Actualmente, los pacientes con NES presentan una mayor tasa de supervivencia y también expectativas de vida mayores. Esto se atribuye tanto al desarrollo de la tecnología, como a la mejora en los cuidados médicos perinatales y adultos. Si bien se estima que la prevalencia de los pacientes con NES ha aumentado, no se cuenta con información específica. El primer estudio nacional de la discapacidad en Chile (ENDISC 2004) ${ }^{3}$ mostró que el $12,9 \%$ de la población presentaba algún tipo de limitación funcional o situación de discapacidad, siendo el 5,7\% de ellos menores de 15 años. Además, se reportó que el 34,6\% de los hogares en Chile tenía al menos un integrante en situación de discapacidad.

Los pacientes con NES presentan un mayor riesgo de desarrollar enfermedades bucales, lo que puede impactar directamente en la salud general y su calidad de vida. La mayoría depende en gran medida de sus cuidadores, quienes no siempre propician una adecuada higiene oral o dieta, por lo que es frecuente encontrar una mayor prevalencia de enfermedad periodontal y caries ${ }^{4}$.
Por otra parte, estos pacientes también presentan una mayor prevalencia de anomalías dento-maxilares, aumento en la severidad de las mismas, y mayor compromiso esqueletal, que en la población normal, lo que dificulta aún más su integración social. La preocupación de los padres y profesionales por el aspecto estético y función normal, ha ido en aumento, siendo el objetivo final lograr la aceptación social, posibilidad de empleabilidad laboral y avanzar hacia la auto-suficiencia. Esto ha generado una creciente demanda por el tratamiento de ortodoncia, sin embargo, continúa siendo uno de los grupos con menores posibilidades de acceder a él 5 .

La presente revisión se enfoca principalmente en aquellas situaciones de discapacidad cognitiva, del desarrollo o física, en la que los individuos generalmente no presentan la habilidad de entender, asumir responsabilidades o cooperar, siendo su atención odontológica todo un desafío ${ }^{6}$. Algunos ejemplos de estas condiciones son el síndrome de Down, la parálisis cerebral y los trastornos del espectro autista.

El objetivo de este trabajo es describir, según la literatura disponible, las consideraciones para el tratamiento ortodóncico en pacientes con necesidades especiales en salud.

\section{Necesidad de tratamiento ortodóncico}

El tratamiento de ortodoncia en los pacientes con NES ha sido cuestionado, principalmente por el riesgo que conlleva y la posibilidad de producir daños iatrogénicos. Sin embargo, como señala Waldman et al. 7 "todo se reduce a la pregunta básica": ¿Creemos que las personas con discapacidades necesitan de consideraciones funcionales y estéticas comparables con aquellas de personas "normales"? Si bien hay requerimientos mínimos para ejecutar un tratamiento de ortodoncia, no se debe desechar la posibilidad solo por el hecho de que un paciente presente algún tipo de discapacidad, ya que éste puede impactar de forma significativa su calidad de vida ${ }^{8}$.

Como se mencionó anteriormente, los pacientes con NES presentan una mayor prevalencia de anomalías dento-maxilares severas, siendo su etiología multifactorial ${ }^{9}$. Entre los factores descritos en la literatura pode- 
mos encontrar: factores hereditarios; mayor presencia de anomalías craneo-faciales que alteran el crecimiento y desarrollo normal del individuo; desarrollo neuromuscular alterado; mayor prevalencia de hábitos parafuncionales como respiración bucal, interposición de lengua u objetos y succión digital ${ }^{10}$; pérdida prematura de dientes temporales, principalmente como consecuencia de una dieta cariogénica (predominantemente blanda y alta en hidratos de carbono) e higiene oral deficiente ${ }^{7,11}$.

Se ha reportado que las maloclusiones severas pueden agravar la situación de un niño con NES, por ejemplo, un overjet muy aumentado puede incrementar la predisposición a traumatismos dentoalveolares y el apiñamiento dentario predisponer a enfermedad periodontal. A su vez, pueden generar problemas funcionales a nivel de la masticación, deglución, salivación, fonación e incluso alteraciones en la articulación témporo-mandibular ${ }^{12}$. Por otra parte, se ha visto que las maloclusiones severas impactan negativamente el aspecto psicosocial de los pacientes con NES, siendo muchas veces causa de una mayor discriminación social y estereotipos negativos ${ }^{13}$.

Respecto al tipo de maloclusiones más frecuentes, generalmente se relacionan con el síndrome o condición del paciente, estableciendo cierto patrón que es importante conocer para poder diagnosticar de forma temprana e instaurar medidas preventivas e interceptivas. A continuación se describen algunos de ellos.

Parálisis cerebral. Corresponde a un grupo de condiciones que se caracterizan principalmente por la presencia de disfunciones motoras, que pueden estar asociadas con un deterioro sensorial o cognitivo, derivado de una lesión cerebral no progresiva durante el desarrollo ${ }^{14}$. Su clasificación depende del tipo de alteración motora predominante, siendo el tipo espástico el más común. Éste se caracteriza por una lesión en la corteza cerebral que produce una hipertonicidad de los músculos faciales. En el tipo atetósico, predominan los movimientos involuntarios y la musculatura facial suele estar hipotónica. Las alteraciones oclusales más frecuentes, son la Clase II molar de Angle, overjet aumentado y mordida abierta anterior ${ }^{15}$, lo que podría explicarse por un sellado labial alterado, una posición adelantada de la lengua junto con movimientos linguales involuntarios, mayor prevalencia de hábitos de succión y respiración bucal ${ }^{16-18}$.

Síndrome de Down. Es un trastorno genético causado por una trisomía del cromosoma 21, caracterizado por la presencia de un grado variable de deterioro cognitivo y rasgos físicos particulares que dan un aspecto reconocible. Estos pacientes presentan mayor riesgo de desarrollar desórdenes que pueden interferir con el crecimiento y desarrollo normal, tales como hipotiroidismo, enfermedad celíaca, defectos cardíacos congénitos, enfermedades autoinmunes y deficiencia de la hormona del crecimiento. Además, pueden presentar anomalías en las relaciones de la base de cráneo, vías aéreas estrechas, hipertrofia tonsilar, posición adelantada de la lengua, musculatura perioral hipotónica y respiración bucal ${ }^{19}$. Esto se manifiesta a nivel dento-maxilo-facial con una mayor prevalencia de hipoplasia maxilar, tendencia a clase III, mordida cruzada, mordida abierta, discrepancias en el tamaño dentario y agenesias ${ }^{20}$.

Andersson et al. ${ }^{19}$ reportaron que las maloclusiones más frecuentes en pacientes de 8 años con síndrome de Down fueron el apiñamiento dentario, mordida cruzada posterior, agenesia de uno o más dientes permanentes y mordida invertida anterior. Asimismo, el 100\% de la muestra presentó necesidad de tratamiento ortodóncico según el Îndice Noruego de Necesidad de Tratamiento Ortodóncico (NOTI por sus siglas en inglés). Otros autores también han reportado una alta prevalencia de dientes retenidos e impactados en este grupo de pacientes ${ }^{20}$.

Trastornos del espectro autista (TEA). Correponde a un grupo de trastornos del desarrollo que se caracterizan por alteraciones en la interacción social y comunicación, junto con patrones de comportamiento estereotipados repititivos y restrictivos. Las maloclusiones más prevalentes en este grupo de pacientes son: overjet severamente aumentado, mordida cruzada posterior y apińamiento dentario maxilar. Estas alteraciones podrían asociarse a una mayor prevalencia de hábitos parafuncionales como el uso de chupete, bruxismo, empuje lingual, succión de labio, comportamientos autolesivos e interposición de objetos, que en el resto de la población ${ }^{21}$.

En relación a la cantidad de pacientes con NES que requieren tratamiento ortodóncico comparada con los que efectivamente lo reciben, existen varias publicaciones. Nayak et al. ${ }^{11}$ evaluaron la prevalencia de la necesidad de tratamiento ortodóncico en 492 pacientes con NES, mayor a 12 años en Dharwad, India. Ellos encontraron que un $50,2 \%$ de los pacientes requería tratamiento ortodóncico, cifra muy elevada al compararla con la población general del mismo rango etario (alrededor de 23\%) ${ }^{22}$, sin embargo, ninguno de ellos presentó tratamiento de ortodoncia. Khursheed et al. ${ }^{23}$ evaluaron la prevalencia de maloclusiones en niños de 7 a 14 ańos con necesidades especiales en Mathura, India, y reportaron que, de los 200 niños examinados, el 70\% presentaba maloclusiones severas y ninguno de ellos se encontraba en algún tipo de tratamiento ortodóncico al momento del examen. Esto da cuenta de la gran necesidad de tratamiento y escaso acceso a la atención que presenta este grupo de pacientes.

Por otra parte, Mohlin y Kurol ${ }^{24,25}$, establecieron que la evaluación de la necesidad de tratamiento no puede basarse solo en índices ortodóncicos, sino más bien depende de las consecuencias de la maloclusión en el paciente y en su calidad de vida.

\section{Motivación para el tratamiento}

¿Cuál es la motivación de los padres para buscar tratamiento ortodóncico? Al-Sarheed et al. ${ }^{26}$ evaluaron las actitudes de los padres de niños con NES hacia el tratamiento de ortodoncia mediante una encuesta. La mayoría se mostró interesado y cooperador, siendo la estética el factor determinante en la búsqueda de tratamiento. No obstante, es frecuente que las familias estén tan involucradas emocional, física y económicamente con la 
condición médica del paciente, que les resulta difícil priorizar un tratamiento ortodóncico, ya que éste no resolverá la discapacidad y supondrá una carga adicional tanto para los padres como para el paciente.

Por otra parte, Becker et al. ${ }^{27}$ establecen que la principal motivación de los padres para buscar tratamiento ortodóncico, es la mejora en la salud y función oral. Cuando los padres y los pacientes suponen que el objetivo final del tratamiento ortodóncico no es lograr la perfección, sino que una mejoría en la estética y función, que podría repercutir positivamente en el aspecto social, no quedan dudas sobre los beneficios del tratamiento, aunque éste conlleve ciertos sacrificios para la familia. Al preguntarles a los padres de los pacientes con NES tratados ortodóncicamente si recomendarían el tratamiento a otros padres, la mayoría respondió que sí, e incluso volverían a repetirlo en sus propios hijos.

\section{Barreras para la atención ortodóncica}

Como ya se ha mencionado anteriormente, los pacientes con NES presentan ciertas barreras para la atención ortodóncica, como las que señalan a continuación:

- Dificultad para el acceso a la atención: grandes costos económicos asociados a los tratamientos de ortodoncia, ausencia o escasa cobertura de programas gubernamentales, falta de conciencia de los padres y/o cuidadores respecto a la necesidad de tratamiento de los pacientes con NES ${ }^{26}$.

- Disposición de los profesionales: factores como poca experiencia, formación académica insuficiente en el área, incapacidad para contar con un equipo multidisciplinario o interés personal, pueden llevar a la elección de no atender a pacientes con NES ${ }^{13,28}$. Un estudio realizado en Estados Unidos y Canadá, reportó que menos del 30\% de los estudiantes de ortodoncia mostró interés en cursar un programa especializado en la atención de pacientes con NES ${ }^{29}$.

- Comportamiento general: es habitual que los pacientes con NES presenten menor capacidad de entendimiento, cortos períodos de atención, tolerancia limitada a ruidos y olores, miedo y ansiedad respecto a situaciones y personas desconocidas, dificultad para tolerar procedimientos simples.

- Movimientos de extremidades y cabeza descontrolados, dificultad para permanecer quieto.

- Reflejo nauseoso exagerado.

- Salivación y "babeo" excesivo ${ }^{30}$.

\section{Principios generales de tratamiento}

El tratamiento ortodóncico en pacientes con NES se encuentra muchas veces justificado, sin embargo, la selección de los pacientes es de suma importancia. En primer lugar, los padres y/o cuidadores deben apoyar por completo el tratamiento y entender a cabalidad el nivel de compromiso necesario para el éxito; esto debe verse reflejado en la mantención de una higiene oral adecuada para prevenir la inflamación gingival o cualquier complicación derivada de ella ${ }^{13}$. El profesional debe educar y motivar constantemente a los padres y, de ser posible, al paciente, sobre una correcta técnica de cepillado. A su vez, los padres y/o cuidadores deben hacerse completamente responsables de la higiene oral del paciente y de su asistencia a los controles ortodóncicos ${ }^{36}$.

En segundo lugar, se recomienda la planificación de un tratatamiento en etapas; donde la primera fase correspondería a la adaptación del paciente a la atención. Esto puede demorar varias sesiones y es importante dedicarle el tiempo necesario para que el paciente adquiera confianza. Existe consenso en la literatura respecto a que una gran parte de los pacientes con NES, son suficientemente cooperadores como para sobrellevar la atención ortodóncica con técnicas simples de manejo conductual como el "decir-mostrar-hacer" y el refuerzo positivo ${ }^{31,36}$.

El uso de sedación profunda o anestesia general, debe ser reservado exclusivamente para procedimientos largos y complejos, como por ejemplo la cementación de brackets, esto siempre y cuando el paciente demuestre la cooperación necesaria para tolerar los controles solo con el uso de técnicas simples de manejo conductual, como se mencionó anteriormente ${ }^{32}$.

En tercer lugar, se debe intentar reducir al mínimo el tiempo de tratamiento correctivo, para lo cual el ortodoncista puede intervenir tempranamente mediante estrategias preventivas, mantención de espacios, erupción dentaria guiada o dirigir el crecimiento a través de dispositivos ortopédicos interceptivos.

Otro aspecto a considerar es que el tratamiento por fases contribuye a la introducción gradual de aparatos en la boca del paciente, lo que puede ayudar al clínico a detectar posibles problemas de cooperación antes de extraer dientes y estar comprometido a cerrar los espacios generados ${ }^{13}$.

Un aspecto importante de la atención ortodóncica en pacientes con NES, es el establecimiento de objetivos de tratamiento realistas. Cuando las condiciones están comprometidas por factores adversos, el tratamiento debe ser redireccionado hacia objetivos acorde a la condición del paciente, muchas veces lejos del ideal, lo que no debe constituir una razón para considerarlo como un fracaso o denegar la atención ${ }^{36}$.

En relación a la modalidad de tratamiento, algunos autores reportan que, la tolerancia de los pacientes con discapacidades intelectuales a los aparatos fijos (multibrackets), es mayor que a los dispositivos removibles, ya que esta técnica permite movimientos dentarios más rápidos y simples ${ }^{33}$. Sin embargo, desde el punto de vista del ortodoncista, los multibrackets son más difíciles de posicionar y requieren de condiciones específicas para su instalación. Además, interfieren de manera considerable con la higiene oral, junto con requerir cooperación adicional de los padres y/o cuidadores cuando se indican elásticos intermaxilares. 
Por otra parte, los dispositivos removibles no interfieren con la higiene oral, y son activados de forma extraoral, evitando cualquier incomodidad al paciente ${ }^{32,36}$.

En conclusión, la modalidad de tratamiento debe ser elegida según los objetivos planificados y el nivel de tolerancia de cada individuo.

Algunos elementos que pueden contribuir a facilitar la atención, es el uso de ciertos avances tecnológicos que han tenido lugar a lo largo del tiempo. Entre ellos, podemos mencionar: el uso de hidrocoloides de gelificación rápida para la toma de impresiones en un menor período de tiempo, disminuyendo las posibilidades de activar el reflejo nauseoso; cementos de vidrio ionómero, que permiten cierta humedad en el ambiente y alambres de alta memoria, que permiten una activacion más espaciada en el tiempo.

Respecto a la longitud del tratamiento y sus resultados, Blanck-Lubarsch et al. ${ }^{34}$ compararon estos aspectos entre pacientes con NES y un grupo control. Ellos reportaron que la longitud general del tratamiento no tuvo diferencias significativas entre ambos grupos, pero sí, el grupo con NES requirió un mayor "tiempo-sillón" que el grupo control, lo que concuerda con lo reportado por Taddei et al. ${ }^{35}$. Respecto a los resultados clínicos obtenidos, éstos fueron inferiores en el grupo estudio, hecho que fue atribuido a una mayor preocupación por parte del ortodoncista y padres, por mejorar el aspecto funcional por sobre el estético, no obstante, todos los pacientes obtuvieron resultados estéticos aceptables.

En cuanto a la fase de retención, ésta adquiere aún mayor importancia en los pacientes con NES, ya que la mayoría de las veces existe un componente esqueletal, etiología neuromuscular y posiciones alteradas de la lengua, que no pueden ser controladas durante y post tratamiento y atentan contra la estabilidad del sistema. Es por esto, que la fase de retención debe ser mantenida por largo período de tiempo. En relación a la modalidad fija o removible, esto se debe decidir tomando en cuenta la cooperación de los padres y/o cuidadores y del paciente, ya que si bien los retenedores removibles son fáciles de instalar, dependen en un 100\% de su uso en el hogar. Los retenedores fijos pueden requerir una sesión extra de sedación para su correcta instalación, pero pueden ser mejor tolerados y no dependen de la cooperación del paciente y su familia ${ }^{32,36}$.

Respecto a las complicaciones del tratamiento, Abeleira et al. ${ }^{32}$ reportaron que la complicación más frecuente en el tratamiento ortodóncico con aparatos fijo (brackets) en pacientes con síndrome de Down, fue la aparición de úlceras orales, aparentemente de origen traumático. Éstas pueden llegar a ser muy extensas y requerir la remoción temporal de los aparatos. Por este motivo, es importante establecer un plan de controles seguidos durante los días iniciales post instalacion de brackets.

\section{Conclusiones}

Los pacientes con NES presentan una mayor prevalencia y severidad de anomalías dento-maxilo-faciales, que afectan tanto la estética como la función de todo el sistema estomatognático, e impactan directamente su salud general y calidad de vida.

El ortodoncista debe reconocer su rol en el equipo multidisciplinario necesario para manejar estos pacientes de forma temprana e integral, contribuyendo a minimizar los efectos deletéreos de las maloclusiones y facilitar su integración social, con el fin último de mejorar su calidad de vida.

La clave para el éxito del tratamiento es la adecuada selección del paciente, considerando sus características anatómicas, fisiológicas y conductuales, junto con el grado de cooperación y motivación de los padres o cuidadores.

\section{Referencias bibliográficas}

1. American Academy of Pediatric Dentistry. Definition of special health care needs. [Consultado el 29 de marzo 2019]. Disponible en: https:/www.aapd.org/research/ oral-health-policies--recommendations/special-health-care-needs/.

2. Instituto Nacional de Estadística. Estadísticas vitales 2012. [Consultado el 29 de marzo 2019]. Disponible en: http://www.ine.cl/canales/menu/publicaciones/calendario_de_publicaciones/pdf/completa_vitales_2012.pdf.

3. ENDISC-CIF Chile 2004. Primer estudio nacional de la discapacidad en Chile. [Consultado 11 Mayo 2019]. Disponible en: https://www.senadis.gob.cl/pag/136/1196/ resultados_endisc_i

4. Dharmani C. Management of children with special health care needs (SHCN) in the dental office. J Med Soc. 2018;32(1):1-6. DOI: 10.4103/jms.jms_115_16.

5. Oreland A, Heijbel J, JagellS. Maloclusions in physycally and/or mentally handicapped children. Swed Dent J. 1987;11(3):103-119.

6. American Academy of Pediatric Dentistry. Symposium on lifetime oral health care for patients with special needs. Pediatr Dent. 2007;29(2):92-152.

7. Waldman HB, Perlman SP, Swerdloff M. Orthodontics and the population with special needs. Am J Orthod Dentofac Orthop. 2000;118(1):14-17. DOI: 10.1067/ mod.2000.105236

8. Hobson RS, Nunn JH, Cozma I. Orthodontic management of orofacial problems in young people with impairments: review of the literature and case reports. Int J Paediatr Dent. 2005;15(5):355-363. DOI: 10.1111/j.1365-263X.2005.00642.x

9. Dinesh RB, Arnitha HM, Munshi AK. Malocclusion and orthodontic treatment need of handicapped individuals in South Canara, India. Int Dent J. 2003;53(1):13-18.

10. Saito K, Jang I, Kubota K, Hoshino T, Hotokezaka H, Yoshida N, Fujiwara T. Removable orthodontic appliance with nickel-titanium spring to reposition the upper incisors in an autistic patient. Spec Care Dentist. 2013;33(1):33-39, DOI: 10.1111/j.17544505.2012.00291.x

11. Nayak PP, Prasad K, Bhat YM. Orthodontic treatment need among special health care needs school children in 
Dharwad, India: A comparative study. J Orthodont Sci 2015;(4):47-51. DOI: 10.4103/2278-0203.156029.

12. Proffit WR. Contemporary orthodontics. 3a ed. Saint Louis: Mosby Inc; 2000. p. 15-17.

13. Rada R, Heba H, Carla E. Orthodontic care for the behavior-challenged special needs patient. Spec Care Dentist. 2015;35(3):138-142. DOI: $10.1111 /$ scd.12082.

14. Gupta R, Appleton RE. Cerebral palsy: not always what it seems. Arch Dis Child 2001;85(5):356-360.

15. Rodrigues dos Santos MT, Masiero D, Novo NF, Simionato MR. Oral conditions in children with cerebral palsy. J Dent Child (Chic). 2003;70(1):40-46.

16. Miamoto CB, Ramos-Jorge ML, Pereiral LJ, Paiva SM, Pordeus IA, Marques LS. Severity of malocclusion in patients with cerebral palsy: determinant factors. Am J Orthod Dentofacial Orthop. 2010;138(4):394.e1-394. e5. DOI: 10.1016/j.ajodo.2010.03.025.

17. De Castilho LS, Abreu MHNG, Pires E Souza LGA, Romualdo LTA, Souza E Silva ME, Resende VLS. Factors associated with anterior open bite in children with developmental disabilities. Spec Care Dentist. 2018;38(1):4650. DOI: $10.1111 /$ scd. 12262 .

18. Yogi H, Alves LAC, Guedes R, Ciamponi AL. Determinant factors of malocclusion in children and adolescents with cerebral palsy. Am J Orthod Dentofacial Orthop. 2018;154(3):405-411. DOI: 10.1016/j.ajodo.2017.11.042.

19. Andersson EM, Axelsson S, Katsaris KP. Malocclusion and the need for orthodontic treatment in 8-year-old children with Down syndrome: a cross-sectional population-based study. Spec Care Dentist. 2016;36(4):194200. DOI: $10.1111 /$ scd.12160.

20. Musich DR. Orthodontic intervention and patients with Down syndrome. Angle Orthod. 2006;76(4):734-735. DOI: 10.1043/0003-3219(2006)076[0734:OIAPWD]2.0. $\mathrm{CO} ; 2$.

21. Fontaine-Sylvestre C, Roy A, Rizkallah J, Dabbagh B, Ferraz Dos Santos B. Prevalence of malocclusion in Canadian children with autism spectrum disorder. Am J Orthod Dentofacial Orthop. 2017;152(1):38-41. DOI: 10.1016/j.ajodo.2017.02.014

22. Bali RK, Mathur VB, Talwar PP, Chanana HB. National oral health survey and fluoride mapping 2002-2003 India. New Delhi: Dental Council of India; 2004.

23. Khursheed O, Gupta S, Sarkar S. Prevalence of malocclusion among 7-14 years old specially abled children attending various special schools in Mathura district, India. J Adv Med Dent Scie Res. 2017;5(4):56-61. DOI: 10.21276/jamdsr.2017.5.4.13.
24. Mohlin B, Kurol J. To what extent do deviations from an ideal occlusion constitute a health risk? Swed Dent J. 2003;27(1):1-10

25. Mohlin B, Kurol J. A critical view of treatment priority indices in orthodontics. Swed Dent J. 2003;27(1):11-21.

26. Al-Sarheed M, Bedi R, Hunt NP. The views and attitudes of parents of children with a sensory impairment towards orthodontic care. Eur J Orthod. 2004;26(1):87-91.

27. Becker A, Shapira J, Chaushu S. Orthodontic treatment for disabled children: motivation, expectation, and satisfaction. Eur J Orthod. 2000;22(2):151-158.

28. Al-Sarheed M, Bedi R, Alkhatib MN, Hunt NP. Dentists' attitudes and practices toward provision of orthodontic treatment for children with visual and hearing impairments. Spec Care Dentist. 2006;26(1):30-36.

29. Noble J, Schroth RJ, Hechter FJ, Huminicki A, Wiltshire WA. Motivations of orthodontic residents in Canada and the United States to treat patients with craniofacial anomalies, cleft lip/palate, and special needs. Cleft Palate Craniofac J. 2012;49(5):596-600. DOI: 10.1597/10-192.

30. Shapira J, Becker A, Moskovitz M. The management of drooling problems in children with neurological dysfunction: a review and case report. Spec Care Dentist. 1999;19(4):181-185.

31. Chaushu S, Becker A. Behaviour management needs for the orthodontic treatment of children with disabilities. Eur J Orthod. 2000;22(2):143-149.

32. Abeleira MT, Pazos E, Limeres J, Outumuro M, Diniz M, Diz P. Fixed multibracket dental therapy has challenges but can be successfully performed in young persons with Down syndrome. Disabil Rehabil. 2016;38(14):13911396. DOI: $10.3109 / 09638288.2015 .1103318$.

33. Chadwick SM, Asher-McDade C. The orthodontic management of patients with profound learning disability. Br J Orthod. 1997;24(2):117-125. DOI: 10.1093/ ortho/24.2.117

34. Blanck-Lubarsch M, Hohoff A, Wiechmann D, Stamm T. Orthodontic treatment of children/adolescents with special health care needs: an analysis of treatment length and clinical outcome. BMC Oral Health. 2014;14:67. DOI: $10.1186 / 1472-6831-14-67$.

35. Taddei M, D'Alessandro G, Amunni F, Piana G. Orthodontic treatment of a particular subgroup of children with special health care needs, children with craniofacial anomalies: An analysis of treatment length and clinical outcome. Angle Orthod. 2016;86(1):115.120. DOI: $10.2319 / 122014.1$

36. BeckerA, ChaushuS, Shapira J. Orthodontictreatment for the special needs child. Semin Orthod. 2004;10(4):281292. DOI: $10.1053 /$ j.sodo.2004.09.009 